\title{
Molecular detection of tick-borne haemopathogens in shelter dogs and Rhipicephalus sanguineus (sensu lato) ticks from Peninsular Malaysia
}

\begin{abstract}
Ticks are important vectors in transmitting various pathogens and they could jeopardize the health and welfare of humans and animals worldwide. The present study aimed to investigate the presence of important tick-borne haemopathogens $(\mathrm{TBH})$ in dogs and ticks via polymerase chain reaction (PCR) assays. A total of 220 blood samples and 140 ticks were collected from 10 animal shelters in Peninsular Malaysia. Of 220 blood samples, 77 (35\%) were positive to TBH, of which $20 \%$ were E. canis, $12 \%$ were A. platys, $7 \%$ were B. gibsoni and $7 \%$ were B. vogeli. All ticks were identified as Rhipicephalus sanguineus with five samples (3.57\%) positive with TBH. Co-infections of TBH (0.45-9.55\%) in dogs were also observed in this study.
\end{abstract}

Keyword: Tick; Shelter dogs; Malaysia; Tick-borne haemopathogens; Vector-borne 